\title{
Fatty acid methyl and ethyl esters as well as wax esters for evaluating the quality of olive oils
}

\author{
Maurus Biedermann • Annette Bongartz • \\ Carlo Mariani $\cdot$ Koni Grob
}

Received: 27 April 2008 / Revised: 22 May 2008 / Accepted: 3 June 2008 / Published online: 19 June 2008

(C) Springer-Verlag 2008

\begin{abstract}
A promising correlation between chemical analysis and sensorial evaluation was confirmed: extra virgin olive oils with low contents of methyl and ethyl esters of fatty acids as well as straight chain wax esters were sensorially evaluated as being of high quality, whereas some with high contents were even devaluated as not being of extra virgin quality. Methanol and ethanol formed during fermentation in degrading olives are esterified, largely by transesterification with fatty acids from the triglycerides, and in this way transferred into the pressed oil. The presence of high contents of methyl and ethyl esters in degrading olives was confirmed. Wax esters from the skin of the olives are extracted at low yields, whereby the yield increases when the olives are soft and possibly degrading. High wax ester contents may, therefore, stand for mild oils, but also for deficient oils.
\end{abstract}

Keywords Olive oil - Ethyl oleate - Wax esters · Sensorial evaluation $\cdot$ Degrading olives

M. Biedermann $\cdot$ K. Grob $(\bowtie)$

Official Food Control Authority of the Canton of Zurich,

P.O. Box, 8032 Zurich, Switzerland

e-mail: Konrad.Grob@klzh.ch; koni@grob.org

A. Bongartz

University of Applied Sciences Zurich (ZHAW),

P.O. Box 335, 8820 Waedenswil, Switzerland

C. Mariani

Stazione Sperimentale per le Industrie degli Oli e dei Grassi,

Via Giuseppe Colombo 79, 20133 Milan, Italy

\section{Introduction}

The quality of olive oils is sensorially tested by elaborate and well established methods (EU regulation 2568/91/EEC and amendments). It enables the classification of the pressed oils into the classes of extra virgin oil, virgin oil and lampant oil [1]. Nonetheless, it would be convenient to have chemical analytical methods for screening oils or supporting sensorial analysis, perhaps even tracing back deficiencies.

A number of approaches were investigated to determine deficiencies of extra virgin olive oils resulting from inappropriate technological treatments, such as spectrophotometry for the determination of conjugated fatty acids, stigmastadiene for the detection of decoloration or high temperature deodoration [2] and the formation of pyropheophytine from chlorophyll [3-6] or the isomerization of 1,2-diglycerides to 1,3-diglycerides $[7,8]$ for the recognition of weak thermal treatments. These methods primarily aimed at detecting adulteration of extra virgin oils by treated oils of minor quality, which does not necessarily affect the sensorial quality. A different approach uses the profile of the flavor components for the characterization of olive oils, e.g., [9].

The content of free fatty acids is used as a quality criterion for extra virgin olive oils. If present at elevated concentrations, free fatty acids indicate lipase activity hydrolyzing triglycerides in the olives, i.e., decay of cell compartmentation. It is a qualification of oil related to the quality of the olives, in contrast to, e.g., peroxide values serving as indicator of oil oxidation.

The straight chain wax esters (long, straight chain fatty alcohols esterified with fatty acids) were shown to be useful quality indicators for olives and oils obtained from these [10-13]. They are located in the waxy surface layer of the 
olive and are poorly extracted by the oil pressed from the fruit. The amount extracted is higher the more soft, possibly degraded the olives are. Typically oils of inferior quality (lampant oils) contain these wax esters at increased concentrations. Solvent (hexane) extracts even higher amounts from the pression residues (pomace), which is used for detecting the admixture of pomace oil to pression oil. A legal limit was established for the wax esters $\mathrm{C}_{40}-\mathrm{C}_{46}$ in extra virgin olive oils $(250 \mathrm{mg} / \mathrm{kg})$ and refined olive oils (350 mg/kg; Commission Regulation 702/2007 amending Regulation 2568/91). However, Mariani [14, 15] repeatedly warned that this limit may be exceeded without such adulteration, if aging of the oil causes free alcohols to be esterified. They may also be formed during raffination, in particular during deodoration.

Cert et al. [16] recently proposed using fatty acid methyl and ethyl esters as quality markers. Methanol and ethanol are formed during fermentation of olives; they cannot be analyzed as such in the oil, since they are largely removed with water during pression, and even the most gentle deodoration would eliminate them. However, they form methyl and ethyl esters of fatty acids, which are transferred into the oil.

Mariani and Bellan [17] took this idea and indeed found a good correlation of the content of these esters with the quality of olive oils determined by other means. They concluded that in extra virgin oil the ratio of methyl to ethyl esters of fatty acids should not be below 0.9-1, i.e., the methyl esters should predominate the ethyl esters. The concentration of ethyl oleate should not exceed $15 \mathrm{mg} / \mathrm{kg}$ : the summed concentrations of the methyl and ethyl oleate not exceed $30-40 \mathrm{mg} / \mathrm{kg}$. Methyl and ethyl oleate can be removed by deodoration at elevated temperatures, but this leaves behind other traces, such as stigmastadiene, and the ratio of the methyl to ethyl esters remains low.

The presence of the methyl and ethyl esters of fatty acids in olive oils was detected long ago. In 1986, Mariani and Fedeli [10] isolated the "nonpolar fraction" of olive oil by means of column liquid chromatography and analyzed it by GC. This method was devised for the broader investigation of the "minor components", such as alcohols, sterols, triterpene alcohols, tocopherols and esters of these, as described in, e.g., $[11,14,18,19]$.

The work described in this paper started out from the online LC-GC-FID method for analyzing minor components $[20,21]$ and the wax ester fraction [13] and optimized it for the combined analysis of methyl/ethyl oleate and selected straight chain wax esters. Extra virgin olive oils from the market were analyzed and those with the highest and lowest concentrations of these markers sensorially tested to check the correlation between the sensorial quality and the analytical data. Additional experiments were performed on the formation of the esters, aiming at clarifying their origin and significance for oil evaluation.

\section{Experimental}

Materials

On-line LC-GC-FID and LC-GC-MS were performed on Thermo Scientific equipment (Milano, Italy), consisting of a TriPlus autosampler, a Phoenix 40 dual syringe pump with three switching valves and a TRACE gas chromatograph equipped with on-column injector, flame ionization detector and a switching valve for the regulation of the transfer. Mass spectrometry (MS) involved a Polaris Q ion trap (Thermo Scientific).

Pentane and methyl tert. butyl ether (MTBE) from Brenntag, Schweizerhall AG (Basel, Switzerland) were redistilled. Heptadecanoic and nonadecanoic acid as well as octadecenol, methyl cis-11,14-eicosadienoate, docosanoyl chloride, the dimethyl polysiloxane PS-255 and triheptadecanoin were from Fluka (Buchs, Switzerland), heneicosanol and ethyl arachidate from Sigma (via Fluka). Edible oils and preserves in cans or jars containing olive oil were from the local market. The wax ester 21-22:0 was prepared as described in [10].

Analytical method

Wax esters were isolated using HPLC in normal phase (NPLC): to $25 \mathrm{mg}$ edible oil weighed into an autosampler vial, $25 \mu \mathrm{l}$ internal standard and verification standard solution was added (esters Me-17:0, Et-20:0, Me-20:2 and 21-22:0, $20 \mu \mathrm{g} / \mathrm{ml}$ in MTBE, corresponding to $20 \mathrm{mg} / \mathrm{kg}$ in the sample) and the vial filled up with $1.5 \mathrm{ml}$ hexane. Of this solution, $10 \mu \mathrm{l}$ was injected into a $250 \times 2 \mathrm{~mm}$ i.d. HPLC column packed with Spherisorb Si $5 \mu \mathrm{m}$ (Grom, Rottenburg-Hailfingen, Germany) and chromatographed with $4 \% \mathrm{MTBE} /$ pentane at $300 \mu \mathrm{l} / \mathrm{min}$. The elution window of the wax ester fraction usually comprised 2-4 $\min$ in retention time, i.e., amounted to $600 \mu \mathrm{l}$. It was adjusted by transferring smaller fractions, including those adjacent to that of interest. The fraction was transferred on-line into GC through the on-column interface by the concurrent eluent evaporation technique. The vapor exit was closed $0.1 \mathrm{~min}$ after the end of the transfer.

The GC system consisted of a $40 \mathrm{~cm} \times 0.53 \mathrm{~mm}$ i.d. precolumn, in the laboratory statically coated with a 0.03 $\mu \mathrm{m}$ film of OV-1701-OH, connected via a T-piece union to a solvent vapor exit and a $20 \mathrm{~m} \times 0.25 \mathrm{~mm}$ i.d. separation column coated in the laboratory with a $0.12-\mu \mathrm{m}$ film of PS255 (a dimethyl polysiloxane). Helium was used as carrier gas at $60 \mathrm{kPa}$ inlet pressure; during transfer pressure was reduced to $40 \mathrm{kPa}$. The oven temperature was initially at $50{ }^{\circ} \mathrm{C}(3 \mathrm{~min})$, then programmed at $30^{\circ} \mathrm{C} / \mathrm{min}$ to $130{ }^{\circ} \mathrm{C}$ and at $7{ }^{\circ} \mathrm{C} / \mathrm{min}$ to $360{ }^{\circ} \mathrm{C}(4 \mathrm{~min})$. 


\section{Sensorial testing}

Sensorial testing was done by the Swiss Olive Oil Panel (SOP) from the University of Applied Sciences Waedenswil (ZHAW). The panel, accredited according to ISO 17025 , consisted of selected and well-trained olive oil experts monitored in accordance to their skills in distinguishing between similar samples by an experienced panel leader. The sensorial evaluation was done by at least 12 panelists according to the EC regulation 796/2002, attachment XII, based on several regulations of the International Olive Council (IOC). The equipment (glass for olive oil tasting, heating device, profile sheet and additional accessories) corresponded to the specifications of the above-mentioned regulations.

The samples, marked with a three digit-code, were presented to the panellists in standardized tasting glasses and tested at a temperature of $28 \pm 2{ }^{\circ} \mathrm{C}$. First, the olfactory stimuli were perceived by smelling the sample after removing the watch-glass from the tasting glass. Second, the oral sensations were tested, such as the overall retronasal olfactory, gustatory and tactile sensations, after taking a sip of about $3 \mathrm{ml}$ oil, well distributed throughout the whole of the mouth cavity. Sensations were documented on a profile sheet.

\section{Results}

Analytical method

The method of analysis was focused on determining methyl and ethyl oleate (Me-18:1 and Et-18:1, the dominant methyl and ethyl esters of fatty acids in olive oil) as well as the wax esters of the $\mathrm{C}_{26}$ and $\mathrm{C}_{28}$ alcohol with unsaturated $\mathrm{C}_{18}$ fatty acids, primarily oleic acid (26-18:X and 28-18:X). These wax esters were selected as markers by Mariani [17] because of negligible interference with diterpene esters).

Figure 1 shows the relevant sections of the LC-GC-FID chromatograms of two extra virgin olive oils. Oil A had a rather high content of the esters of interest, oil $\mathrm{B}$ a low one. The fatty acid methyl and ethyl esters are eluted early, followed by the dominant squalene. The waxes in the second half of the chromatogram start with the diterpene esters, first of all the phytol esters and minor geranylgeraniol esters originating from the pulp of the olive [13]. These are followed by the straight chain wax esters, originating from the skin of the olive. They are well visible in oil A, but small in oil B. The chromatogram ends with the sterol esters of fatty acids.

The method of analysis was derived from that used for the analysis of the wax esters [13]. HPLC in normal phase was used to isolate the esters of interest, and the

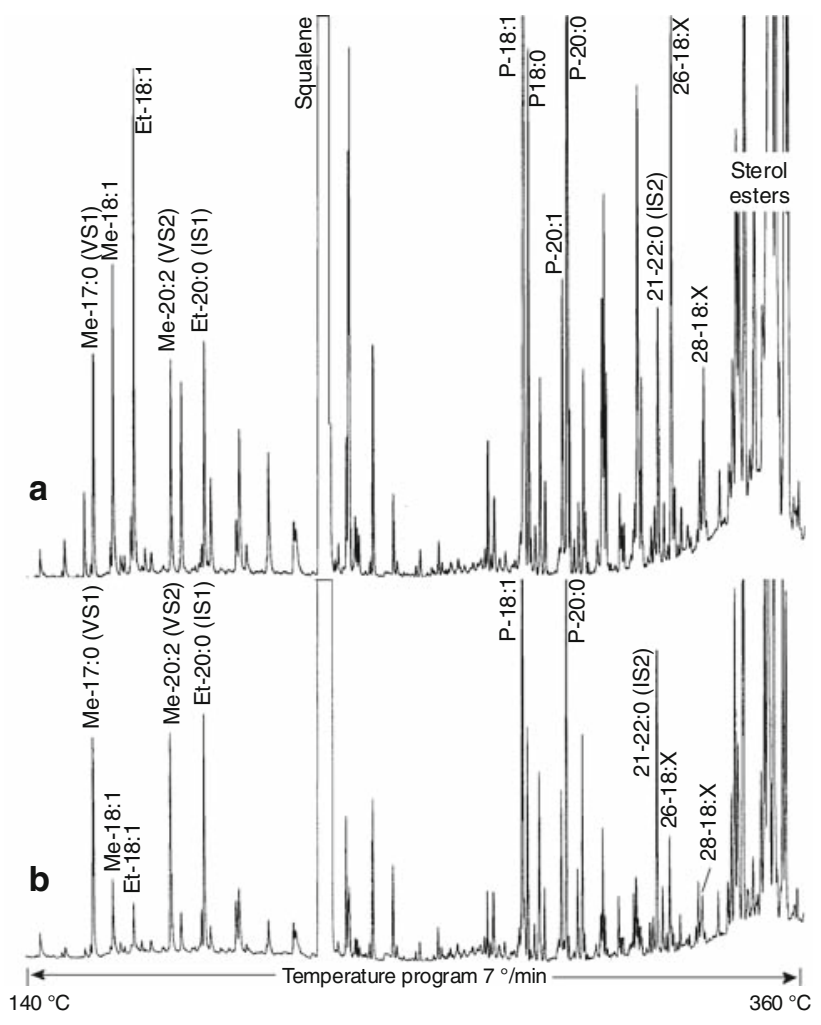

Fig. 1 LC-GC-FID chromatograms of the esters of interest; a extra virgin oil with rather high concentrations of methyl and ethyl oleate and straight chain wax esters; $\mathbf{b}$ extra virgin oil with low concentrations of these components. IS internal standard; VS verification standard. Labeling of esters: alcohol-fatty acid, for acids with carbon number:number of double bonds (written out in the text)

corresponding fraction was transferred on-line to GC-FID, enabling automatic analysis of diluted oils with the internal standards.

For the transfer of the HPLC fraction into GC, the retention gap technique is used if volatile constituents are of interest: solvent trapping retains these during eluent evaporation in the uncoated precolumn [22]. Fully concurrent eluent evaporation can be used if no solvent trapping is needed. Since this requires just a short precolumn and adjustment of conditions is usually uncritical, it is preferred whenever applicable. Initially, concurrent eluent evaporation was performed with the loop-type interface [22]. Later, the transfer by the on-column interface was preferred, as it enables to do with a single interface and to apply the same techniques as also used for large volume on-column injection [23].

Fatty acid methyl esters are among the most volatile compounds that can be transferred with concurrent eluent evaporation without losses, provided the evaporation conditions are selected with some care. It means that the eluent should evaporate in the inlet of the precolumn at a temperature little above the dew point of the carrier gas/vapor mixture. This temperature is below the eluent boiling point and is the lower the higher is the dilution of the vapors with 
carrier gas, i.e., the higher is the ratio of the gas flow rate/ eluent transfer rate. Optimization occurred by a mixture containing $\mathrm{C}_{10}-\mathrm{C}_{28} n$-alkanes at equal concentrations or empirically by lowering the oven temperature during transfer until breakthrough is noticed by loss of solutes (usually being drastic): at too low temperatures, incomplete eluent evaporation causes liquid to accumulate in the precolumn, and since the capacity of the latter to retain liquid is small, the carrier gas discharges most of the liquid through the solvent vapor exit.

Quantitative analysis was based on two internal standards, namely, ethyl eicosanoate (Et-20:0; IS1 in Fig. 1) for determining methyl and ethyl oleate and the wax ester 2122:0 (IS2) for the esters 26:18:X and 28-18:X. FID response was assumed to be equal, i.e., no correction for the response was applied. The detection limits of the analysis are determined by interferences and, therefore, depend on the oil. Usually they were around $1.5 \mathrm{mg} / \mathrm{kg}$ for the oleates and $3 \mathrm{mg} / \mathrm{kg}$ for the wax esters.

The method was conceived on the basis of checking the critical points by verification [24], i.e., with tools built into the method to control adequate performance of each analysis. Using concurrent eluent evaporation, loss of fatty acid esters by co-evaporation with the eluent is a critical point. The internal standard used for calculation (Et-20:0) was less volatile than the methyl and ethyl oleate. A more volatile verification standard was added, methyl heptadecanoate (Me-17:0; VS1), which would be more affected than the analytes and the internal standard; a potential loss would have been recognized by a reduced ratio of $\mathrm{Me}-17: 0 / \mathrm{Et}-$ 20:0. Results were accepted if Me-17:0/Et-20:0 exceeded 0.95 of the value calibrated in the mixture of standards by on-column injection.

Another critical point concerned the HPLC fraction window; a shift in retention times may cause incomplete transfer to GC. For its control, a verification standard was used on each edge of the fraction. Figure 2 shows the elution order from NPLC for an extra virgin olive oil with an intermediate ester content. On top, the LC-GC-FID chromatogram corresponding to the total fraction is shown, i.e., from 2 to 4 min LC retention time; below, 30 s slices are shown. The fraction prior to the chosen window (1:30-2:00 min) was virtually empty. The first $30 \mathrm{~s}$ of the transferred fraction (2:00-2:30 min) included breakthrough, as shown by the presence of the $n$-alkanes (dominated by $n-\mathrm{C}_{29}$ ). It also included the (strongly overloading) squalene and part of the wax esters. The cut at 2:30 min separated the wax esters and shows that the unsaturated species are somewhat more retained than the saturated ones. In particular, the internal standard 21-22:0 is in the first fraction to a larger extent than the two wax esters analyzed. This is why it was chosen not only for calculating the wax esters, but also for the control of the beginning of the fraction; losses would be accentuated and visible by a decrease of the 21-22:0/Et20:0 ratio.

The fatty acid esters are eluted after the wax esters, whereby the ethyl esters were in the window 3:00-3:30 min and the methyl esters in the last 30-s section. The bottom chromatogram $(4: 00-4: 30 \mathrm{~min})$ confirms that at 4:00 min the elution of the solutes of interest was complete. For verification of the cut at the end of the fraction, methyl eicosadienoate (Me-20:2; VS2) was added; as a methyl ester with two double bonds it is eluted somewhat later than the solutes of interest and IS1 (Et-20:0). Its complete inclusion into the fraction was checked by the Me-20:2/Et-20:0 ratio. Hence, totally four standards were added and three area ratios calculated for each analysis to assure adequate performance of the analysis.

The complete separation of the fatty acid methyl and ethyl esters from the wax esters observed in Fig. 2 shows a potentially interesting option; if only the methyl/ethyl esters were of interest, a narrow fraction situated after the wax and sterol esters could be transferred to GC, enabling to stop the $\mathrm{GC}$ run at around $250{ }^{\circ} \mathrm{C}$.

Table 1 shows data on the reproducibility of the results for extra virgin oil with moderately high ester contents. For six consecutive injections, absolute peak areas varied with a relative standard deviation (RSD) of up to $3.5 \%$. The RSDs for the concentrations calculated from the internal standards reached $1.3 \%$. It confirms that on-line LC-GC-FID is a highly reproducible technique as long as the critical parameters are under control.

The measuring uncertainty of the method depends on the accuracy of preparing the solutions, uncertainty about the relative responses of the FID and accuracy of peak integration. When chromatographic interference is minor, this uncertainty is below $10 \%$.

\section{Evaluation of olive oils from the market}

Forty olive oils from the Swiss market sold as extra virgin were analyzed chemically and a selection of these also sensorially. The columns at the left in Table 2 present the concentrations of the esters of interest. Samples are tabulated by increasing concentration of ethyl oleate, i.e., by decreasing quality as regards this parameter. The next column shows the ratio of the methyl/ethyl oleate and the formula proposed by Mariani [17], which is the concentration of ethyl oleate multiplied to the sum of the wax esters 26-18:X and 28-18:X.

The columns at the right in Table 2 characterize the sensorial quality for the seven oils with the lowest and highest ethyl oleate contents as well as for some oils of more particular interest. The evaluation was performed according to the EC regulation 796/2002, attachment XII. The method classifies virgin olive oils according to the intensity of 
Fig. 2 LC-GC-FID chromatograms of an extra virgin olive oil: complete fraction (top chromatogram) as well as $30 \mathrm{~s}$ slices of the transferred fraction and the adjacent windows confirming the absence of material of interest outside the fraction analyzed. $I S$ internal standard; $V S$ verification standard

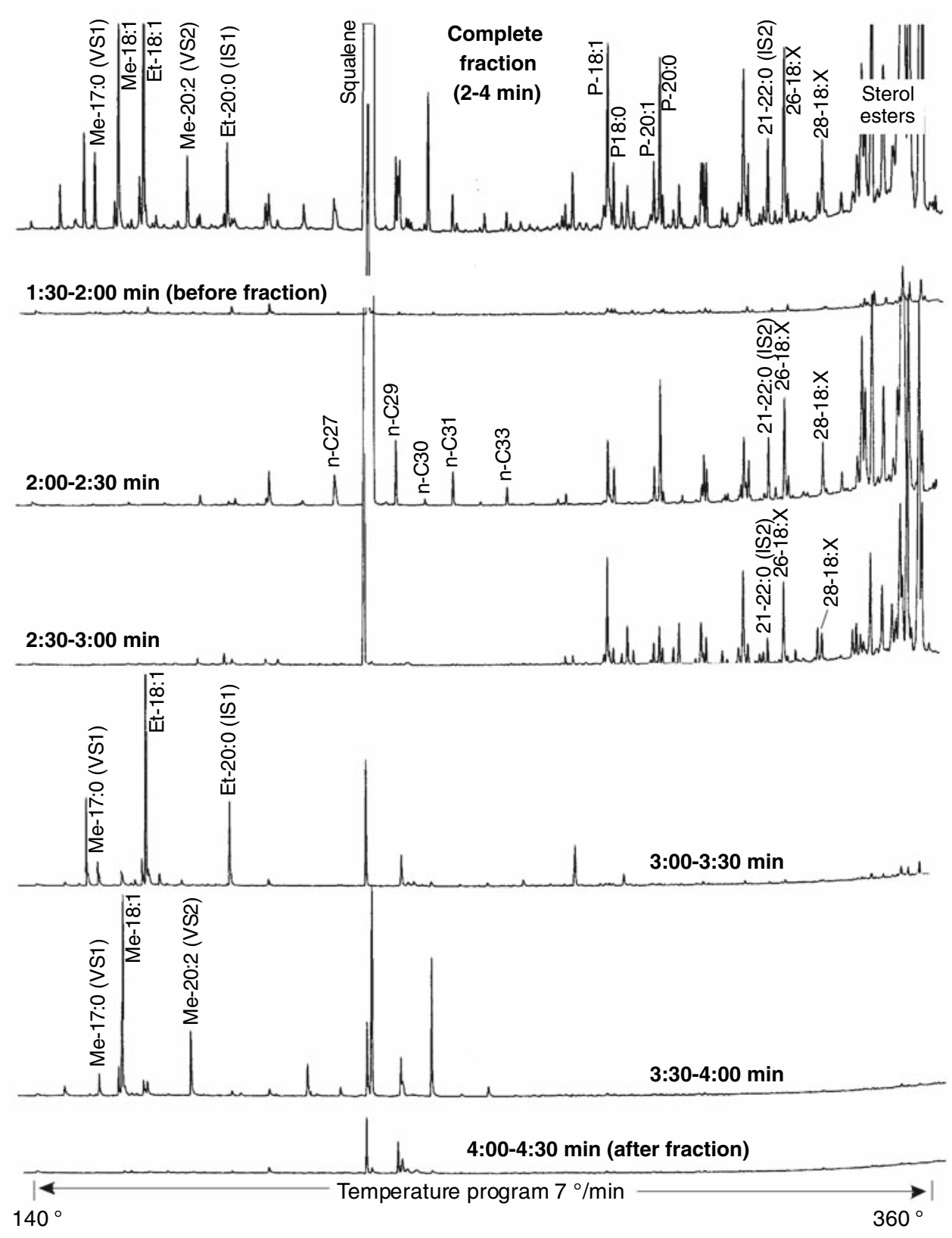

defects perceived and evaluates the fruitiness. The attribute "harmony" was tested according to a method validated for the "Testing Laboratory for Consumer Tests and Sensory Analysis of Food" at the University of Applied Sciences Zurich. It is described as positive sensory impression, reflecting the degree of balance of all positive characteristics, taking into account all olfactory, tactile and kinaesthetic stimuli.

According to the previous work [17], the best oils were low in contents of fatty acid ethyl esters, and the fatty acid methyl esters dominated the ethyl esters, i.e., the concentration ratio of methyl oleate/ethyl oleate was above 1 . The wax ester concentrations were low, indicative of firm and hard olives. Indeed, the sensorial qualification of the oils low in ethyl esters (oils 1-7) was high. The oils tended to be bitter and pungent (green), as to be expected from fresh olives. Also oils 15, 16 and 18 were favorably evaluated in the sensorial test, well fitting the analytical data.

Oil 20 was characterized by a clearly increased Et-18:1 concentration $(6.2 \mathrm{mg} / \mathrm{kg})$, but Me-18:1 was even higher $(18 \mathrm{mg} / \mathrm{kg})$, still resulting in a high ratio of $\mathrm{Me}-18: 1 / \mathrm{Et}-$ 18:1. Also the wax ester concentration was clearly higher $(28 \mathrm{mg} / \mathrm{kg})$, well corresponding to the sensorial evaluation as light and mature. There was no relevant sensorial deficiency, corresponding to expectations for oil obtained from ripe and still intact olives. The chemical data of oil 23 was similar. The sensorial quality was taxed rather high, the lower wax ester content going along with a stronger taste. 
Table 1 Repeatability of data obtained from six consecutive injections of an extra virgin olive oil

\begin{tabular}{lcc}
\hline & Mean & RSDs \\
\hline \multicolumn{2}{l}{ Absolute peak areas } \\
Me-18:1 & 1,858 & 2.7 \\
Et-18:1 & 3,026 & 3.1 \\
Et-20:0 & 1,611 & 2.4 \\
E44 & 4,919 & 3.5 \\
E46 & 1,617 & 3.5 \\
Concentrations (mg/kg) \\
Me-18:1 & 23.1 & 0.6 \\
Et-18:1 & 37.6 & 1.1 \\
E44 & 42.0 & 1.2 \\
E46 & 13.8 & 1.3 \\
\hline
\end{tabular}

The oil 30 was characterized by a clearly increased Et18:1 with a Me-18:1/Et-18:1 ratio of 0.2 only. The wax ester content was low. The sensorial evaluation did not properly correspond to this; the oil was considered light, without deficiency and of a rather high overall quality. The compositional data from oil 34 was similar to oil 30, again rather high in Et-18:1 and low in the Me-18:1/Et-18:1 ratio, with an intermediate wax ester content, nevertheless evaluated of mild and harmonious quality.

The sensorially poor oils were at the bottom of the table. Oil 35 was among the highest in Me-18:1 (60 mg/kg) and with an increased Et-18:1 concentration $(30 \mathrm{mg} / \mathrm{kg})$, but still a high Me-18:1/Et-18:1 ratio (2.1). As the wax esters were also high $(45 \mathrm{mg} / \mathrm{kg})$, this resulted in a high value for the product of the ethyl and wax esters (1,300; Mariani [17] suggested a limit of 300 for a high quality oil). The oil was considered light and only of lampant quality. Oil 38 contained $53 \mathrm{mg} / \mathrm{kg}$ Et-18:1 combined with about half as much methyl ester and high wax ester content, suggesting overripe and fermented olives. The sensorial evaluation confirmed this by a mild and deficient quality. Oil 39 had poor analytical characteristics which were confirmed by the sensorial evaluation. The oil with the highest Et-18:1 concentration and a low Me-18:1/Et-18:1 ratio (nr. 40) contained a modest concentration of wax esters and was sensorially devaluated to the level of virgin oil.

The correlation between the analytical data and the sensorial evaluation was remarkable, taking into account that probably most oils consisted of mixtures and that the low quality components might have undergone some corrective processing (such as deodoration) to remove disturbing deficiencies. In fact, oil 39, the product of lowest quality, also contained $0.14 \mathrm{mg} / \mathrm{kg}$ stigmastadiene, indicating some (illegal) treatment.

Figure 3 plots the concentrations of the methyl oleate against those of the ethyl oleate in the same oils. The inserted line represents a Me-18:1/Et-18:1 ratio of 1 , suggested as a limit between the higher quality oils below the line and the deficient oils above it. In 19 of the 40 oils, the Me-18:1/Et-18:1 ratio was below 1. The ratio varied from 0.26 to 9.2 . The correlation between the methyl and ethyl oleate was weak $\left(R^{2}=0.65\right)$.

Figure 4 plots the sum of the straight chain wax esters of the palmitic and the unsaturated $\mathrm{C}_{18}$ acids with $\mathrm{C}_{22}-\mathrm{C}_{28}$ alcohols against the sum of the methyl and ethyl oleate. The wax esters were analyzed by LC-GC-MS [13]. The correlation is marginal $\left(R^{2}=0.107\right)$, suggesting that the two types of quality markers have little common background. A high wax ester content in an oil is indicative of soft and ripe, but not necessarily deficient olives, whereas the fatty acid methyl and ethyl esters originate from alcohols formed by fermentation, which is not necessarily linked with ripe or overripe olives. Further parameters might interfere, such as the conditions of oil production (malaxation time and temperature or extraction technique influencing the extraction of the wax esters), and degradation not necessarily resulting in fermentation and formation of ethanol. Finally, in some oils the fatty acid methyl and ethyl esters might have been reduced by deodoration.

Three refined olive oils were added to this data set. The concentration of the straight chain wax esters was higher than for all extra virgin oils, as expected for oils from olives of inferior quality. However, the concentration in methyl/ ethyl oleate was low. This is probably the result of deodoration having removed a large proportion of the methyl and ethyl oleate. The Me-18:1/Et-18:1 ratio varied between 0.13 and 0.24 . Assuming that the methyl and the ethyl oleate were removed to a similar extent, these ratios still reflect a strong prevalence of the ethyl ester in the oil, i.e., an inferior quality of the olives and the raw oil submitted to raffination.

Figure 5 plots the straight chain wax ester tetracosanyl palmitate (24-16:0) against the tetracosanyl esters of the unsaturated $\mathrm{C}_{18}$ acids (24-18:X). In high quality extra virgin olive oil, the concentrations are low (filled squares for the 40 commercial oils described above). The four oils devaluated by sensorial testing as lampant or virgin are shown by empty squares and are situated at the upper end of the extra virgin oils, close to the three raffinates. The wax ester concentrations in the olive oils used in preserves, such as vegetables or fish in oil (canned or in glass jars), are in the same range or even above those of the raffinates sold in bottles. Six from the seven oils in preserves declared as extra virgin contained wax esters at the high end of the extra virgin oils sold in bottles.

Fresh versus degraded olives

Fresh and partially degraded olives from the same tree were ground and the oil isolated in the laboratory by centrifuge after malaxation during $1 \mathrm{~h}$. Figure 6 shows a sample of 
Table 2 Chemical and sensorial analysis of 40 olive oils sold as extra virgin

\begin{tabular}{|c|c|c|c|c|c|c|c|c|c|c|c|c|}
\hline & \multicolumn{6}{|c|}{ Chemical analysis } & \multicolumn{6}{|c|}{ Sensorial test } \\
\hline & \multicolumn{4}{|c|}{ Concentrations (mg/kg) } & \multicolumn{2}{|c|}{ Calculation } & \multicolumn{5}{|c|}{ Test results } & \multirow[t]{2}{*}{ Verdict } \\
\hline & Me-18:1 & Et-18:1 & 26-18:X & 28-18:X & $\mathrm{Me} / \mathrm{Et}$ & $\mathrm{Et} \times \mathrm{WE}$ & Fruity & Fruitiness & Bitter & Pungent & Harmony & \\
\hline 1 & 2.7 & 0.3 & 3.1 & 0.6 & 9.2 & 1 & 3.38 & Medium & 3 & 3.45 & 5.54 & Ex. virgin \\
\hline 2 & 1.5 & 0.5 & 6.8 & 1.4 & 2.7 & 4 & 3.83 & Medium & 2.85 & 3.05 & 6.34 & Ex. virgin \\
\hline 3 & 1.2 & 0.8 & 5.3 & 1.1 & 1.6 & 5 & 5 & Intense & 4.6 & 3.9 & 7.14 & Ex. virgin \\
\hline 4 & 1.6 & 1.1 & 2.3 & 1.6 & 1.5 & 4 & 2.65 & Light & 1.5 & 1.9 & 4.96 & Ex. virgin \\
\hline 5 & 1.8 & 1.2 & 6.3 & 1.3 & 1.6 & 9 & 3.98 & Medium & 3.7 & 3.1 & 4.97 & Ex. virgin \\
\hline 6 & 1.6 & 1.2 & 3.8 & 0.8 & 1.4 & 6 & 3.7 & Medium & 2.8 & 2.5 & 6.67 & Ex. virgin \\
\hline 7 & 2.6 & 1.5 & 1.4 & 1.4 & 1.8 & 4 & 3.5 & Medium & 3.7 & 1.5 & 6.51 & Ex. virgin \\
\hline 8 & 1.4 & 1.7 & 5.0 & 2.5 & 0.8 & 12 & & & & & & \\
\hline 9 & 4.9 & 1.8 & 9.1 & 3.4 & 2.7 & 23 & & & & & & \\
\hline 10 & 1.8 & 1.9 & 2.8 & 0.6 & 1.0 & 6 & & & & & & \\
\hline 11 & 5.4 & 2.4 & 17.7 & 7.8 & 2.2 & 62 & & & & & & \\
\hline 12 & 2.2 & 2.8 & 5.3 & 3.3 & 0.8 & 24 & & & & & & \\
\hline 13 & 3.3 & 3.1 & 2.8 & 3.3 & 1.1 & 19 & & & & & & \\
\hline 14 & 4.7 & 3.1 & 7.5 & 3.4 & 1.5 & 34 & & & & & & \\
\hline 15 & 3.4 & 3.1 & 8.3 & 3.2 & 1.1 & 36 & 3.35 & Medium & 2.5 & 3.4 & 5.4 & Ex. virgin \\
\hline 16 & 4.2 & 3.4 & 1.5 & 0.6 & 1.2 & 7 & 2.75 & Light & 3.4 & 2.3 & 3.35 & Ex. virgin \\
\hline 17 & 3.3 & 3.5 & 3.3 & 0.9 & 0.9 & 15 & & & & & & \\
\hline 18 & 4.7 & 3.9 & 3.2 & 2.1 & 1.2 & 21 & 3.8 & Medium & 2.3 & 3 & 5.66 & Ex. virgin \\
\hline 19 & 4.6 & 4.6 & 2.6 & 2.6 & 1.0 & 25 & & & & & & \\
\hline 20 & 17.8 & 6.2 & 20.6 & 6.9 & 2.9 & 169 & 2.6 & Light & 1.6 & 2.2 & 3.94 & Ex. virgin \\
\hline 21 & 7.3 & 6.3 & 5.4 & 1.5 & 1.2 & 44 & & & & & & \\
\hline 22 & 21.8 & 7.2 & 16.9 & 4.8 & 3.0 & 158 & & & & & & \\
\hline 23 & 16.6 & 7.8 & 10.9 & 3.4 & 2.1 & 111 & 3.2 & Medium & 2.1 & 2.7 & 5.83 & Ex. virgin \\
\hline 24 & 6.8 & 9.4 & 7.1 & 3.3 & 0.7 & 98 & & & & & & \\
\hline 25 & 8.2 & 9.6 & 6.1 & 1.8 & 0.9 & 76 & & & & & & \\
\hline 26 & 4.1 & 10.0 & 5.3 & 2.6 & 0.4 & 79 & & & & & & \\
\hline 27 & 9.9 & 12.5 & 3.5 & 1.4 & 0.8 & 61 & & & & & & \\
\hline 28 & 8.5 & 13.9 & 12.3 & 3.9 & 0.6 & 225 & & & & & & \\
\hline 29 & 9.1 & 14.1 & 8.1 & 1.7 & 0.6 & 139 & & & & & & \\
\hline 30 & 4.0 & 16.9 & 2.5 & 2.2 & 0.2 & 80 & 2.1 & Light & 1.2 & 1.7 & 4.3 & Ex. virgin \\
\hline 31 & 25.0 & 21.8 & 28.0 & 10.6 & 1.1 & 843 & & & & & & \\
\hline 32 & 24.7 & 21.9 & 30.3 & 14.7 & 1.1 & 983 & & & & & & \\
\hline 33 & 19.0 & 23.8 & 11.0 & 3.3 & 0.8 & 340 & & & & & & \\
\hline 34 & 14.8 & 28.1 & 10.8 & 4.5 & 0.5 & 428 & 2.75 & Light & 1.5 & 2 & 5.09 & Ex. virgin \\
\hline 35 & 59.6 & 29.1 & 33.2 & 11.7 & 2.1 & 1,306 & 1 & Light & 1 & 1 & 0.97 & Lampant \\
\hline 36 & 11.5 & 35.5 & 1.9 & 3.8 & 0.3 & 201 & & & & & & \\
\hline 37 & 23.4 & 38.9 & 25.3 & 7.6 & 0.6 & 1,281 & & & & & & \\
\hline 38 & 28.7 & 53.4 & 46.0 & 15.6 & 0.5 & 3,289 & 2 & Light & 1 & 1.3 & 0.7 & Lampant \\
\hline 39 & 68.0 & 125.0 & 36.1 & 17.0 & 0.5 & 6,646 & 1.58 & Light & 0.7 & 0.75 & 0.33 & Lampant \\
\hline 40 & 44.5 & 131.2 & 12.4 & 3.5 & 0.3 & 2,078 & 2.03 & Light & 1.4 & 1.4 & 1.13 & Virgin \\
\hline
\end{tabular}

Calculated: ratio Me-18:1/Et-18:1; formula Mariani $($ Et $\times$ WE), Et-18:1 $\times(26-18: X+28-18: X)$

each of these olive lots. The olives at the left have no significant damage, whereas for those at the right at least an addle spot was observed. Some parts became soft and wizen.
The wax esters, analyzed by LC-GC-MS, were grouped in straight chain palmitates (16:0), straight chain esters of unsaturated $\mathrm{C}_{18}$ acids $(18: \mathrm{X})$, as well as phytol $(\mathrm{P})$, geranylgeraniol $(\mathrm{G})$ and benzyl $(\mathrm{Bz})$ esters. The strongly 


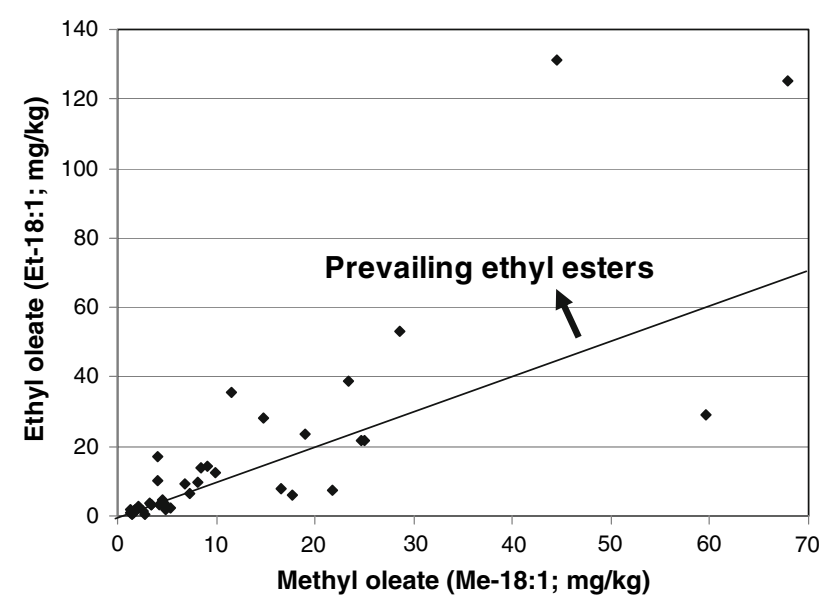

Fig. 3 Concentrations of methyl oleate plotted against those of ethyl oleate in 40 olive oils sold as extra virgin. A ratio of 1 was suggested as a limit for quality evaluation [17], i.e., values above the line are suggested to be indicative of deficient olive oils

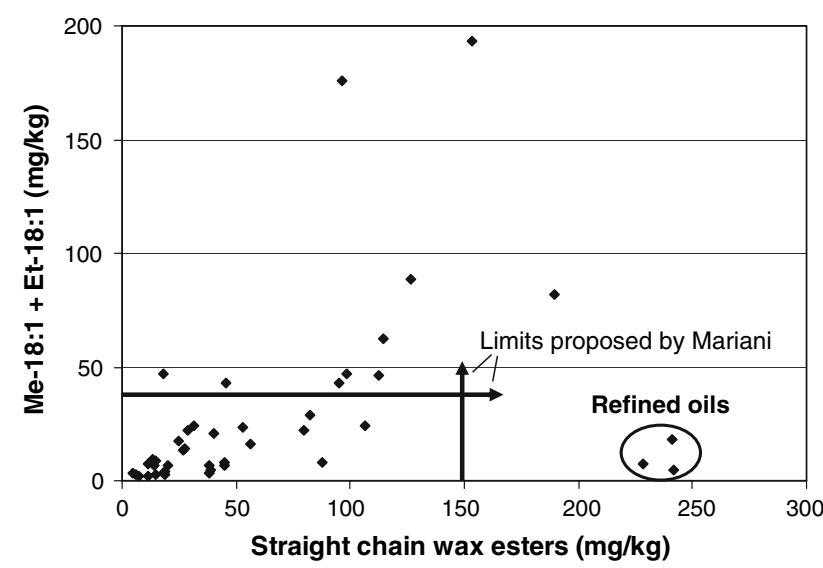

Fig. 4 Summed concentrations of the straight chain wax esters with $\mathrm{C}_{22}-\mathrm{C}_{28}$ alcohols plotted against the sum of the methyl and ethyl oleate in 40 olive oils sold as extra virgin quality and three refined oils; limits suggested by Mariani [17]

predominating phytol esters are presented after reduction by a factor of 5 . At the right, the methyl and the ethyl oleate are added.

Figure 7 shows that 3.8 and 4.8 times more $16: 0$ and the 18:X straight chain wax esters, respectively, were extracted from the somewhat degraded fruits. With $37 \mathrm{mg} / \mathrm{kg}$, the concentration of the wax esters was rather high even for the non-damaged olives (it would have ranked 34 in the 40 extra virgin oils of Table 2) and probably resulted from the several weeks of storage between harvest and analysis. The difference for the diterpene esters was small; they are located in the pulp and anyway well extracted by the oil. It was somewhat larger for the two benzyl esters of the $\mathrm{C}_{26}$ and $\mathrm{C}_{28}$ alcohols.

The degradation caused the concentration of methyl oleate to increase by a factor of 43 , from 0.6 to $28 \mathrm{mg} / \mathrm{kg}$, that

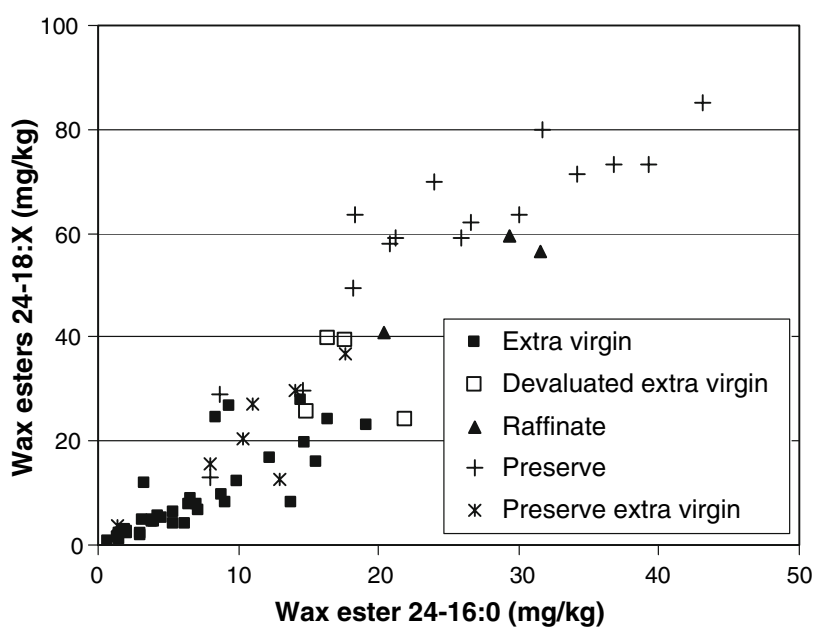

Fig. 5 Wax ester 24-16:0 against the wax esters 24-18:X for various types of olive oils

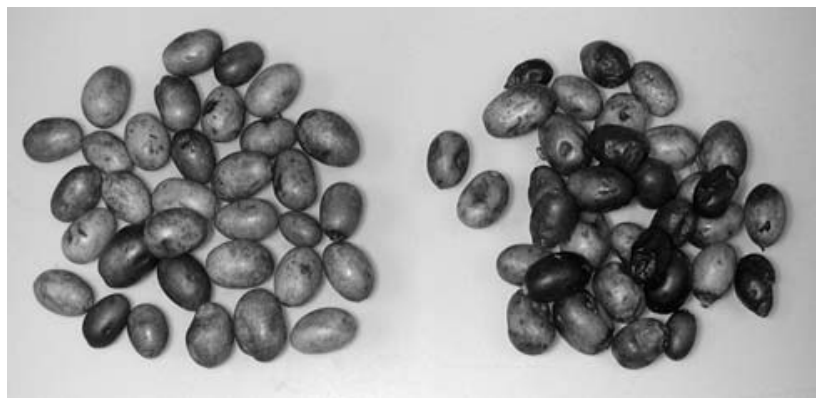

Fig. 6 Photographs of some olives the oil of which was analyzed for methyl and ethyl oleate as well as wax esters. Left olives in good shape; right olives with at least some minor damage

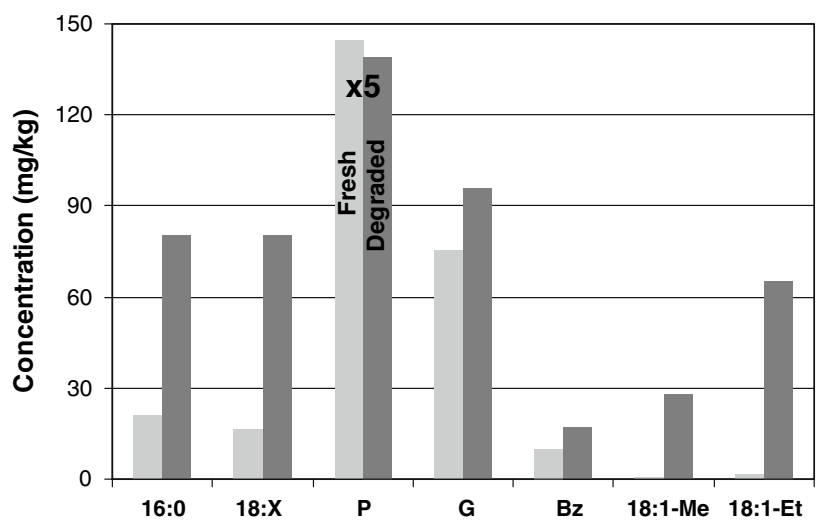

Fig. 7 Esters in the oils from the healthy and partially degraded olives shown in Fig. 6. 16:0 and 18:X, wax esters of palmitic acid and unsaturated $\mathrm{C} 18$ fatty acids, respectively, with $\mathrm{C}_{22}-\mathrm{C}_{28}$ alcohols; $\mathrm{P}$ and $\mathrm{G}$ : wax esters of phytol and geranylgeraniol with the fatty acids $\mathrm{C}_{16}-\mathrm{C}_{26}$; Bz: benzyl esters of $\mathrm{C}_{26}$ and $\mathrm{C}_{28}$ fatty acid. Phytol esters presented after a reduction by a factor of 5

of ethyl oleate by a factor of 50 from 1.3 to $65 \mathrm{mg} / \mathrm{kg}$. This confirms that healthy olives contain hardly any methyl or ethyl esters and that these drastically increase in the oil from damaged fruits. The data also shows that a rather high 
Table 3 Experiments concerning the formation of the esters: additions to a refined olive oil or olive homogenate and formation of the related esters (concentrations in $\mathrm{mg} / \mathrm{kg}$ )

\begin{tabular}{|c|c|c|c|c|c|c|c|c|c|c|c|c|}
\hline & Experiment & Treatment & Me-18:1 & Et-18:1 & $18: 1-\operatorname{Pr}$ & Me-17:0 & Et-17:0 & $\operatorname{Pr}-17: 0$ & 21-18:1 & $21-17: 0$ & $18: 1-18: 1$ & 18:1-19:0 \\
\hline 1 & Oil & $48 \mathrm{~h} / 55^{\circ} \mathrm{C}$ & 6 & 8 & & & & & & & & \\
\hline 2 & $\begin{array}{l}+1 \% \mathrm{FA} 17+1 \% \mathrm{EtOH} \\
+1 \% \mathrm{MeOH}\end{array}$ & $48 \mathrm{~h} / 55^{\circ} \mathrm{C}$ & 600 & 220 & & 18 & 10 & & & & & \\
\hline 3 & $+1 \%$ FA17 & $11 \mathrm{~d} / 40{ }^{\circ} \mathrm{C}$ & 5 & 9 & & $<1$ & $<1$ & & & & & \\
\hline 4 & $\begin{array}{l}+1 \% 21-\mathrm{OH}+0.1 \% \mathrm{MeOH} \\
+0.1 \% \mathrm{EtOH}\end{array}$ & $11 \mathrm{~d} / 40^{\circ} \mathrm{C}$ & 95 & 50 & & & & & 300 & & & \\
\hline 5 & same $+2 \%$ FA17 & $11 \mathrm{~d} / 40^{\circ} \mathrm{C}$ & 650 & 350 & & 35 & 20 & & 1,300 & 120 & & \\
\hline 6 & $+10 \% \operatorname{tri}-17+0.1 \% \mathrm{MeOH}$ & $11 \mathrm{~d} / 60{ }^{\circ} \mathrm{C}$ & 370 & 8 & & 60 & & & & & & \\
\hline 7 & $\begin{array}{l}+1 \% \mathrm{FA} 19+0.1 \% \mathrm{MeOH} \\
\quad+1 \% 18: 1 \mathrm{OH}\end{array}$ & $11 \mathrm{~d} / 40^{\circ} \mathrm{C}$ & 500 & 12 & & 25 & $<1$ & & & & 900 & 40 \\
\hline 8 & $\begin{array}{l}\text { Olive }+1 \% \text { FA17 }+1 \% \text { 21-O } \\
\quad+0.1 \% \text { PrOH }\end{array}$ & $5 \mathrm{~d} / 40{ }^{\circ} \mathrm{C}$ & - & 11 & 220 & & & 45 & 2,200 & 650 & & \\
\hline
\end{tabular}

wax ester content may be linked with low methyl and ethyl esters of fatty acids, provided ripe or aged olives are not damaged.

\section{Formation of the esters}

The ester formation was investigated to gain information about the significance of these quality markers. In particular it was of interest to determine the relative importance of esterification with free acids and transesterification with fatty acids primarily from triglycerides; if esterification is the main route, the methyl and ethyl oleate concentrations reflect the products of the concentrations of the alcohols and free fatty acids in the olives or the oil, whereas in the case of transesterification the free acids interfere at most indirectly.

For the experiment, a refined olive oil (low acidity) with 6 and $8 \mathrm{mg} / \mathrm{kg} \mathrm{Me}-18: 1$ and Et-18:1, respectively, was chosen. A control experiment warming the oil at $55{ }^{\circ} \mathrm{C}$ for $48 \mathrm{~h}$ had no effect on these esters (line 1 in Table 3 ). To this oil, $1 \%(\mathrm{w} / \mathrm{w})$ heptadecanoic acid (FA17) and $1 \%$ each of ethanol and methanol were added (line 2). After warming at $55^{\circ} \mathrm{C}$ for $48 \mathrm{~h}, 600$ and $220 \mathrm{mg} / \mathrm{kg}$ of Me-18:1 and Et-18:1, respectively, were determined, next to 18 and $10 \mathrm{mg} / \mathrm{kg}$ Me-17:0 and Et-17:0, respectively. Since the concentration of the free oleic acid in this oil must have been substantially below that of the added FA17, virtually all Me-18:1 and Et18:1 was formed by transesterification with oleic acid from the triglycerides. Considering that 33 and 22 times less Me17:0 and Et-17:0, respectively, were formed than Me-18:1 and Et-18:1, whereas some 70 times less FA17 was available compared to oleic acid in the triglycerides, esterification was slightly faster than transesterification.

Subsequent experiments were performed with warming to $40{ }^{\circ} \mathrm{C}$ during $11 \mathrm{~d}$. Short initial heating to $70{ }^{\circ} \mathrm{C}$ was necessary to dissolve the added components, particularly the acid. The addition of $1 \%$ FA17 to the refined olive oil had no significant effect (line 3). To a parallel sample, $1 \%$ of the alcohol $\mathrm{C}_{21}$ and $0.1 \%$ each of methanol and ethanol were added. After warming, these alcohols were converted to esters with a yield of roughly $1 \%$. The same experiment was also performed with the addition of 1\% FA17 (line 5). This acid accelerated the transesterification by a factor of between $4.3(21-\mathrm{OH})$ and 7 (ethanol). In addition, small amounts of the esterification products with FA17 were found (in agreement with the experiment of line 2). This shows that free fatty acids promote ester formation, but primarily by acceleration of transesterification, rather than by own esterification.

A further experiment was performed at $60{ }^{\circ} \mathrm{C}$ to prevent re-crystallization of the $10 \%$ triheptadecanoin (tri-17) added together with $0.1 \%$ methanol. Some six times more Me-18:1 than Me-17:0 was found, as expected from transesterification and the abundance of the fatty acids in the triglycerides present in the mixture.

The experiment in line 7 involved the addition of $1 \%$ FA19 and 1\% octadecenol (18:1-OH). Some 23 times more ester with the oleic acid (18:1-18:1) was formed by transesterification than ester 18:1-19:0 by esterification with FA19, confirming that the wax esters formed by aging of olive oils are largely products of transesterification.

The last experiment aimed at testing the effect of enzymes in a homogenate of fresh olives. To this paste, $1 \%$ FA17, $1 \% 21-\mathrm{OH}$ and $0.1 \%$ propanol (virtually absent even in degrading olives) were admixed, added as ten times more concentrated solution in the oil used for the previous experiments. After 5 days at $40{ }^{\circ} \mathrm{C}$, shortly before being grown over by mold, the paste was extracted with hexane and the oil analyzed. Five times more propanol was converted to the oleate than to the heptadecanoate, and substantially more $21-\mathrm{OH}$ reacted by transesterification with oleic acid than by esterification with FA17 (line 8). Comparison with the experiment in line 5 suggests that there was more esterification, but this could have been the result 
of factors other than enzyme activity, such as the presence of other, stronger acids (see also the higher concentration of 21-18:1 than in line 5 despite the shorter incubation).

It is concluded that esterification is slightly faster than transesterification, but that the far higher concentration of esterified acids causes transesterification to be the principal route of ester formation with alcohols. This transesterification is catalyzed by the presence of acid. It means that the methyl and ethyl oleate observed in raw olive oils primarily reflect the presence of the alcohols (indicator of fermentation). Free fatty acids (resulting from lipase activity) and possibly other acids present in the olives contribute to the oleates only indirectly. At a concentration of $1 \%$, fatty acid accelerated transesterification by a factor of 6-7, but catalysis in the olives is not necessarily comparable to our experiment, since stronger acids may be present.

During 11 days at $40{ }^{\circ} \mathrm{C}$, some $5 \%$ of the added alcohol was esterified in an oil containing $1 \%$ free fatty acid. In the olive paste, $4.5 \%$ of the added propanol was converted in 5 days at $40{ }^{\circ} \mathrm{C}$. If the esters were exclusively formed in the fermenting olives, it would follow that $0.1 \%$ alcohol is sufficient to form several $100 \mathrm{mg} / \mathrm{kg}$ esters in a few days. Pression of the oil eliminates most of the alcohols with the water, but during the far longer storage time of the oil even a small amount of alcohol is sufficient to significantly add to the ester content.

The formation of wax esters during storage of oils [14, 15] well fits into these results; as the long chain alcohols are not removed from the oil by the water, there is abundantly time for their esterification, whereby transesterification during storage of oils of a high acidity prior to raffination should be particularly rapid.

\section{Conclusions}

The results confirm that the methyl and ethyl esters of fatty acids are useful indicators for determining the quality of olives and the oil produced from these [17]; sensorial evaluation showed that the oils with low concentration of these esters were of high quality, whereas some of those with the highest contents not even satisfied the minimum quality required for extra virgin oils. As confirmed by oil obtained from degraded olives, these esters originate from the degradation process, presumably fermentation.

Also the straight chain wax esters are indicators for the quality of olive oils, but their background is different. Their concentration in the pressed oil is a function of the extraction yield: the skin of soft olives is extracted more efficiently than that of hard and fresh fruits. This is not necessarily linked with deficiencies; ripe olives releasing substantial amounts of straight chain wax esters may yield mild oils of high quality. However, ripe and soft olives easily change over to degradation, and the skin of degrading olives tends to be soft. The use of straight chain wax esters as quality indicator is further complicated by their continuous formation during storage, which means that the content in the oil is also a function of the long chain alcohols present in the freshly pressed oil and the storage time, which is probably not related to sensorial quality.

In conclusion, methyl and ethyl esters of fatty acids are direct indicators of olive degradation, whereas high wax ester contents are a likely consequence of degrading olives, but not a conclusive indicator of degradation.

\section{References}

1. International Olive Council (COI; 2006). Trade standards applying to olive oils and olive pomace oils. COI/T15/NC No. 3 Rev.2. http://www.internationaloliveoil.org/downloads/NORMAEN1.pdf

2. Lanzon A, Cert A, Albi T (1989) Grasas y Aceites 40:385-388

3. Aitzetmüller K (1989) Proceedings Eurolipids, Angers (F) 1:177

4. Mordret F, Coustille JL, Lacoste F (1997) OCL 4:364-368

5. Serani A, Piacenti D (2001) Riv Ital Sost Grasse 78:459-464

6. Gertz C, Fiebig HJ (2006) Eur J Lipid Sci Technol 108:1062-1065

7. Amelotti G, Daghetta A, Ferrario A (1989) Riv Ital Sost Grasse 66:681-692

8. Pérez-Camino MC, Moreda W, Cert A (2001) J Agric Food Chem 49:699-704

9. Lopez-Feria S, Cardenas S, Garcia-Mesa JA, Valcarcel M (2008) J Chromatogr A 1188:308-313

10. Mariani C, Fedeli E (1986) Riv Ital Sost Grasse 63:3-17

11. Grob K, Lanfranchi M, Mariani C (1990) J Am Oil Chem Soc 67:626-634

12. Bianchi G, Tava A, Vlahov G, Pozzi N (1994) J Am Oil Chem Soc 71:365-369

13. Biedermann M, Haase-Aschoff P, Grob K (2008) Eur Lipid Res Technol (in press)

14. Mariani C, Fedeli E, Grob K, Artho A (1991) Riv Ital Sost Grasse 68:179-187

15. Mariani C, Venturini S (1996) Riv Ital Sost Grasse 73:489-497

16. Cert A (2006), Communication at meeting of International Olive Oil Council, Madrid, 1-4 October 2006

17. Mariani C, Bellan G (2008) Riv Ital Sost Grasse 85:3-20

18. Mariani C, Fedeli E (1989) Riv Ital Sost Grasse 66:397-401

19. Mariani C, Venturini S (2002) Riv Ital Sost Grasse 79:49-57

20. Artho A, Grob K, Mariani C (1993) Fat Sci Technol 95:176-180

21. Grob K, Giuffré AM, Leuzzi U, Mincione B (1994) Fat Sci Technol 96:286-290

22. Grob K (1991) On-Line Coupled LC-GC. Hüthig, Heidelberg. ISBN 3-7785-1872-0.

23. Grob K (2000) J Chromatogr A 892:407-420

24. Grob K (2007) J Chromatogr A 1150:93-99 